\title{
Design of a Novel Type Micro-Stirrer Excited by Longitudinal Elastic Wave for Thrombus Dissolution*
}

\author{
Ajoudanian MOHAMMAD**, Zhongwei JIANG** and Minoru MORITA** \\ **Department of Mechanical Engineering, Yamaguchi University, \\ 2-16-1, Tokiwadai, Ube, 755-8611, Japan. \\ E-mail: jiang@yamaguchi-u.ac.jp
}

\begin{abstract}
This paper presents the method to design a novel micro stirrer for high effective dissolution of cerebral thrombus or blood clot. The micro stirrer is a beam with supported one side and the other side is attached to an asymmetrical stirrer. The waves, which were excited by the transducer, propagate through the catheter and impinge to the inclined surface in the stirrer. The reflected waves generate bending mode in the stirrer to dissolve the thrombus. Tip trajectory results are investigated for the simultaneous estimation of all the mode shapes, due to obtain the best drive frequency. It seems that the expected motion is a mode shape which, despite has the bending at tip, there is no significant bending across the catheter. An important parameter has been developed to act as an indicator factor for mode shape detection in simulation analysis. The design algorithm is offered based on the stirrer length and full length of micro stirrer. The accuracy of the estimated design method is evaluated through the some other stirrer length.
\end{abstract}

Key words: Micro Stirrer, Thrombus, Mode Shape, Catheter, Longitudinal Mode, Bending Mode

\section{Introduction}

Recently serious disability and death causes by cerebral stroke. It could occur due to various reasons. The major amongst them is caused by block age of a cerebral artery by a clot forming in it (thrombus) which interrupts the oxygen and nutrients to part of the brain and induces a brain cell necrosis.

The clot-busting blood protease tissue plasminogen activator (tPA) is used to dissolve the clot and unblock the artery lately. However, the drugs most commonly used in treatment of stroke inducing neuronal damage and causing hemorrhagic stroke ${ }^{(1),(2)}$. The tPA can be executed about three hours after the first symptoms of stroke appear, but a few patient reach to hospital to get this drug on time. Therefore, a quick dissolution of thrombus is required. There were some attempts for mechanical thrombectomy for instance removal of the offending thrombus directly by Merci Retriever ${ }^{(3),}{ }^{(4)}$. It is a catheter employed a corkscrew-like device to ensnare the clot. But it can be effect or injure the blood vessel wall. In the other work, the controlled piezoelectric motion of a fiber, chiral polymer, was used to Grasp and remove the thrombosis ${ }^{(5)}$. Also some other researcher using an ultrasonic catheter device to stimulate the thrombus for a quick recanalization (6) or using a transcranial doppler stimulator to assist the dissolution effect ${ }^{(7)}$. However, these methods induced cavitations and heat in the blood. So it may also be caused damage the brain cell and tissue by collapsing bubbles.

One of the other methods to remove the thrombus is the dissolution of the clot by

*Received 10 May, 2011 (No. 11-0280) [DOI: 10.1299/jbse.6.262]

Copyright $(\odot 2011$ by JSME 
stirring of a beam which excited by mechanical vibration ${ }^{(8)}$. The properties of thrombus are beyond this paper and we'll not focus on it. But it is known that all clots show significant variation of mechanical properties because they made up under different polymerization circumstances ${ }^{(9)}$. Due to this fact the stirrer have to applying different forces, amplitudes and velocities (frequencies) to dissolve the thrombus effectively and immediately. Regarding to previous study ${ }^{(10)}$, it is necessary to create stable and reliable bending motion with certain amplitude of vibration. In some case of clot, the input power or force must be increased to prevent the decreasing of the amplitude. So depends on the thrombus, the required force can be adjust by the size of transducer or input voltage.

In the previous research, the stirring motion was created by using tip deflection of bending actuator such as piezoelectric bimorph or unimorph cantilever type ${ }^{(11)}$. The most effective design parameters for tuning an adaptive stirring are the size of transducer and the tip deflection of the bending actuator. The tip deflection also depends on the total length of the bending actuator. If this bending actuator is supposed to use in the blood vessel, which is smaller than 1-2 $\mathrm{mm}$ in diameter, the required motion by the cantilever type stirrer will be difficult to be achieved. The same problem could also happen for the size of transducer to create required force. In addition, because the transducer is also in the body, increasing the input voltage is restricted.

To overcome these problems, a new idea was proposed ${ }^{(12)}$, in which a powerful transducer is set out of the body so that the required power or force can be easily adjust by the size of transducer and there is no concern for limitations to blood vessels in design. The transducer generates a longitudinal elastic wave in the micro-stirrer and after the longitudinal wave is transmitted to the tip part, it is converted to a flexure wave to excite the bending motion just at the tip part. Unlike the previous type the bending motion isn't created along the total length of micro-stirrer and there is no concern to use in the blood vessel that is smaller than $1-2 \mathrm{~mm}$ in diameter. How to convert efficiently a longitudinal wave to a bending motion only occurred at the tip part is a key problem in design of this type micro stirrer. In this paper, the most important parameters to achieve the bending motion are mentioned first and how to find the optimal parameters is investigated in detail. Finally, an optimal parameter searching algorithm with the aid of numerical simulation is proposed and the algorithm is confirmed by applying in some design examples for the validation of its availability.

\section{Concept}

This intervention care is accomplished by using the catheter with two thin tubes to insert a main flexible tube (Fig. 1). The main tube was advanced over pre-inserted guide wire into the femoral artery. Directing the stirrer into the clot at the cerebral circulation by guide wire, the transducer generates a longitudinal elastic wave and it is transmitted to the stirrer through the catheter. Afterwards, the stirrer excites the clot with also injecting the thrombolytic agent through the thin tube. Finally, the thrombus fragments of the clot are evacuated by vacuum through the other thin tube.

The Fig. 2 presents the concept of micro stirrer for exciting thrombolysis. It contains three major parts, the transducer, catheter and stirrer. The first part at the left end is the piezoelectric transducer which converts electrical power to mechanical vibration. The amplitude of the vibration generated directly by the piezoelectric actuator is very small so that the solid acoustic horn to amplify the elastic wave was designed and provided (Fig. 1). In order to simplify the simulation, just a piezoelectric transducer with the poling direction parallel to the length was employed (Fig. 2). It works as follows. First a five-cycle windowed sinusoid voltage at the required frequency is applied to the transducer to generate the elastic wave at the cross-section of the catheter. 

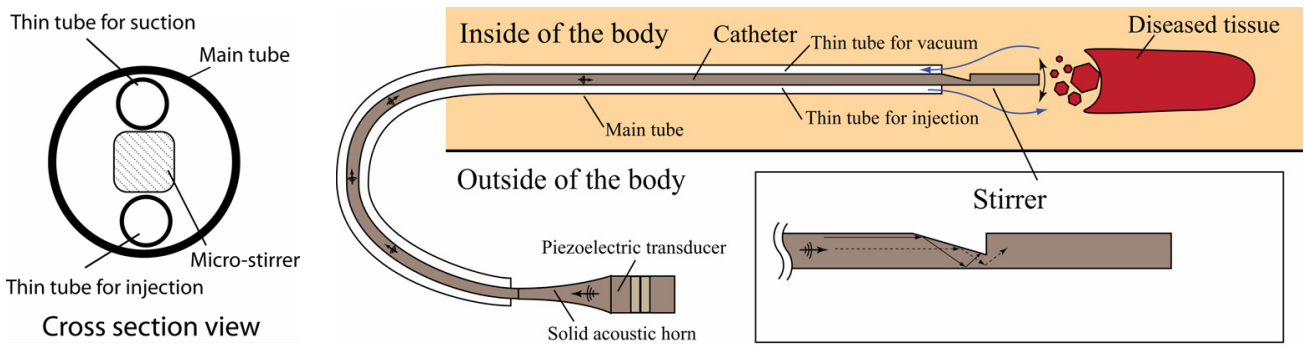

Fig.1 Concept view of the idea to dissolve the thrombus.

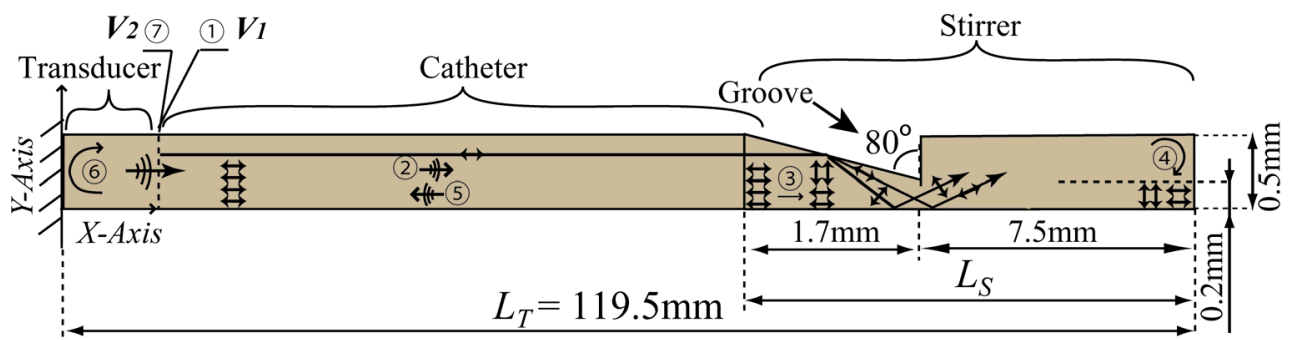

Fig.2 Detail view of micro stirrer, (1)input signal, (2)longitudinal wave propagating to tip, (3)mode conversion, (4)swing stirrer and reflecting back, (5)reflecting wave to transducer, (6impinging and reflecting, (7)next input signal synchronizing with previous reflected input signal. $\leftrightarrow$ : Longitudinal wave, $\mathfrak{I}$ : Shear wave.

After the waves propagate through the catheter into the stirrer, the waves impinge on an interface at an oblique angle and the fluctuating motion in stirrer has occurred due to the reflection and mode conversion caused by the interface.

\section{Effective Drive Method for Micro Stirrer}

In order to find the most effective way to convert the longitudinal wave to the fluctuating motion at the stirrer, a modal analysis with a harmonic simulation was performed to check the modal shapes and to reveal the maximum amplitude at stirrer tip. The FEM modeling was carried out by a commercial FEM software package, ANSYS 10, where the displacement at the left end of the actuator was fixed and all other boundaries were free (Fig. 2).

The material of the beam in following simulation is titanium and the material properties are set as follows: Young's modulus $E=115.7 \mathrm{GPa}$, Density $\rho=4540 \mathrm{Kg} / \mathrm{m} 3$ and Poisson's ratio $v=0.321$. The electric field applied to the piezoelectric stack is $1 \mathrm{kV}$. The element types used for the meshing of the PZT model is SOLID 226 (Coupled-Field Hexahedral) and for catheter and stirrer is SOLID 186. All the mesh size is given by $0.5 \mu \mathrm{m}$.

Fig. 3 exhibits the tip vertical displacement of the stirrer derived from harmonic analysis. In order to evaluate the mode shape of micro stirrer the mode shapes of the catheter and stirrer at three resonant frequencies are illustrated in Fig. 4. The $d_{s}$ and $d_{c}$ denote the maximum vertical deflection at stirrer and catheter, respectively (Fig. 4).

The most significant difference between the mode shapes is the motion on the catheter. In contrast with two other mode shapes, just longitudinal motion has been created in the catheter at $30700 \mathrm{~Hz}$. In another word, there is no significantly vertical deflection occurred on the catheter, which indicates that less energy might be lost in catheter during wave propagation.

As mentioned in the concept, the catheter is contacting with the two thin tubes and also inserted in the main tube (Fig. 1). The tubes or the blood vessel may become the constraint on the vertical movement or the bending motion of the catheter. 


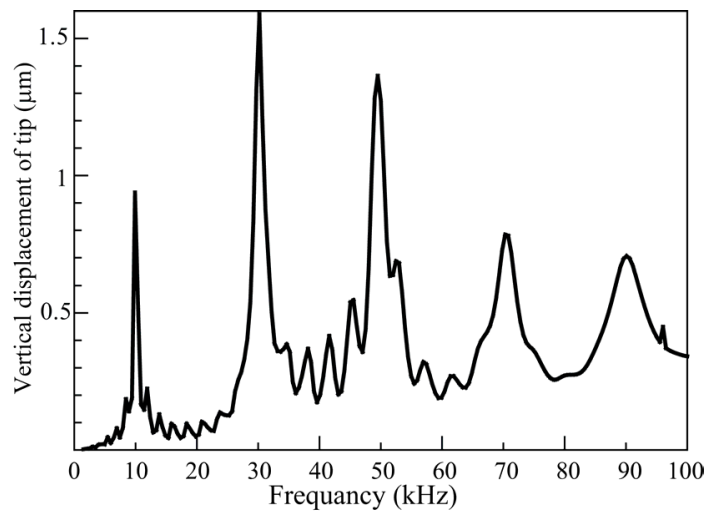

Fig.3 Tip displacement of the stirrer at the resonant frequencies.

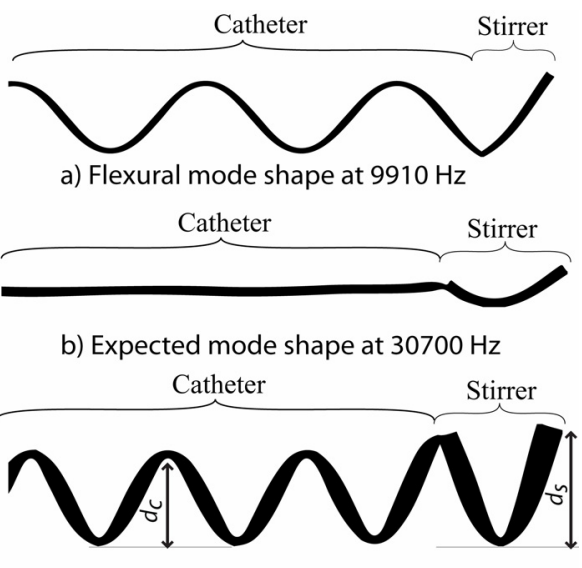

c) Flexural mode shape at $49510 \mathrm{~Hz}$

Fig.4 The vertical motion of micro stirrer derived from modal analysis.

In order to investigate the effect of the catheter on the stirrer motion when the catheter is constrained by the tubes or the vessel, the transient analysis was done. To effectuate this purpose, firstly the tip trajectory of the stirrer is simulated in the boundary condition where the catheter and stirrer were free. Next some mass less springs and dampers on the nodes of catheter to restrict movement in the Y-direction are applied to simulate the constraint condition. Furthermore, the influence of the thrombus on the nodes of stirrer is replaced with dampers in both direction $\mathrm{X}$ and $\mathrm{Y}$ axis.

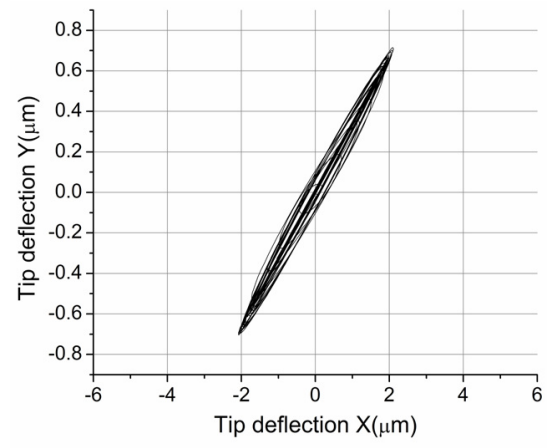

a. Excited by $30700 \mathrm{~Hz}$

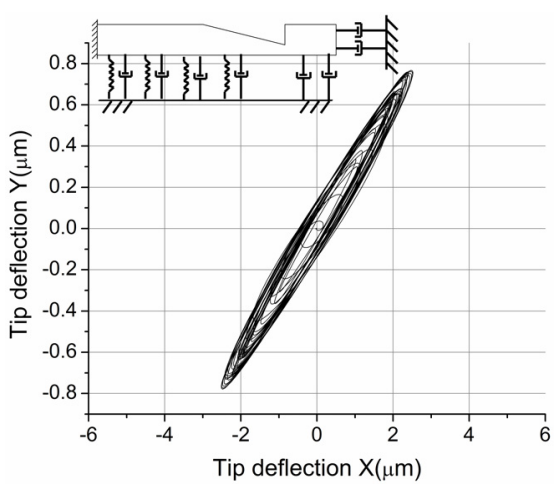

c. Excited by $30700 \mathrm{~Hz}$

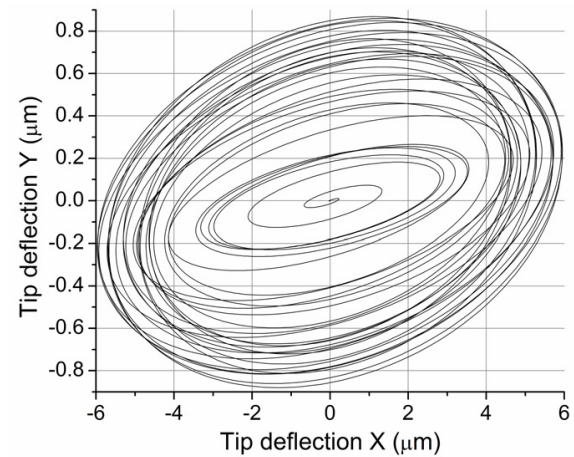

b. Excited by $9910 \mathrm{~Hz}$

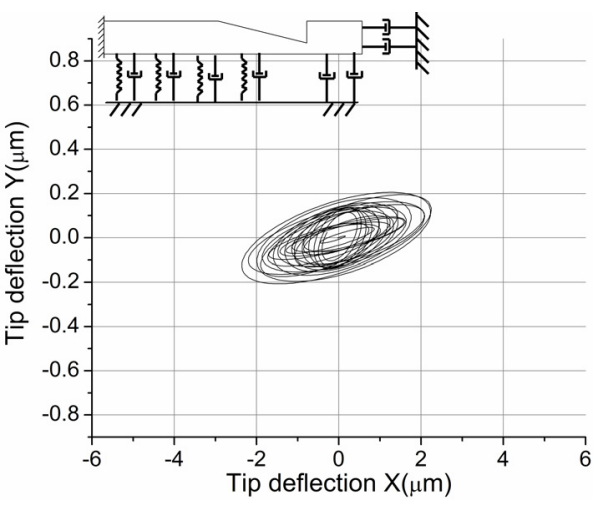

d. Excited by $9910 \mathrm{HZ}$

Fig.5 Trajectory of tip deflection while; a, b) stirrer and catheter assumed as free condition c, d) dampers and springs on catheter and dampers on Stirrer.

The tip trajectory results of stirrer obtained for $9910 \mathrm{~Hz}$ excitation frequency are illustrated in Figs. 5 (b) and (d). As is evident, the tip amplitudes in both X and Y directions 
decrease up to seventy percent in $\mathrm{X}$ and eighty percent in $\mathrm{Y}$ direction when the catheter is under the constraint.

However, there is no significant difference observed between the tip trajectories when the stirrer is excited at $30700 \mathrm{~Hz}$. Also, the mentioned dampers on the stirrer part apply force to the stirrer due to vibration. But the stable amplitude at $30700 \mathrm{~Hz}$ indicates that the stirrer can overpower the damping forces of thrombus which are assumed as a sample. Therefore, with this mode shape the tip motion of the stirrer will be more stable even when the constraint condition is changed (Figs. 5 (a) and (c)).

From the above results and discussions, an ideal case is to obtain the stable bending motion in stirrer and an axial motion in catheter while no vertically motion occurred. However, this ideal case appears just at a special criterion as shown in Fig. 4 (b). The question comes here, how can one design or find this ideal case so that the longitudinal wave transmits in the catheter without or less fluctuation in vertical direction and the bending motion only occurred in the stirrer.

In order to describe this ideal case in simulation analysis, it is essential to define an evaluation index parameter. In fact, this ideal case is occurred at an expected mode shape where the maximum vertical deflection at catheter $\left(d_{c}\right)$ should be negligible small as compared to the deflection at stirrer $\left(d_{s}\right)$. So the ratio of these maximum vertical deflections is defined in Eq. (1), called as indicator expected mode shape ratio (IEM ratio). It is used to evaluate the expected mode shape quantitatively in simulation analysis.

$$
I E M=\frac{\text { Maximum vertical deflection at stirrer }\left(d_{\mathrm{s}}\right)}{\text { Maximum vertical deflection at catheter }\left(d_{\mathrm{c}}\right)}
$$

The high value of the ratio usually describes a high efficient wave energy transmission. On the other hand, it gives more restriction in determination of the design parameters.

Fig. 6 illustrates the IEM ratios as a function of the stirrer length $\left(L_{s}\right)$ while the groove angle is fixed to $80^{\circ}$. The IEM ratios are obtained when aforementioned three frequencies (Fig. 4) are used to excite the stirrer. It is evident that the IEM ratio at $30700 \mathrm{~Hz}$ is much higher than the other two modes, and it becomes the highest value when the length of stirrer is $9.2 \mathrm{~mm}$. If the value of IEM ratio is set higher than 10 , the stirrer length at $L_{S}=9.2 \mathrm{~mm}$ and excitation frequency at $30700 \mathrm{~Hz}$ will be the optimum parameters to achieve the ideal result. However, the optimum length of stirrer $L_{s}=9.2 \mathrm{~mm}$ is strongly related to the total length $L_{\mathrm{T}}=119.5 \mathrm{~mm}$.

Now consider a practical application case. Suppose the stirrer length $L_{S}$ is given due to the application purpose, how can one find an optimum total length $L_{\mathrm{T}}$ ? The detail will be discussed in next section.

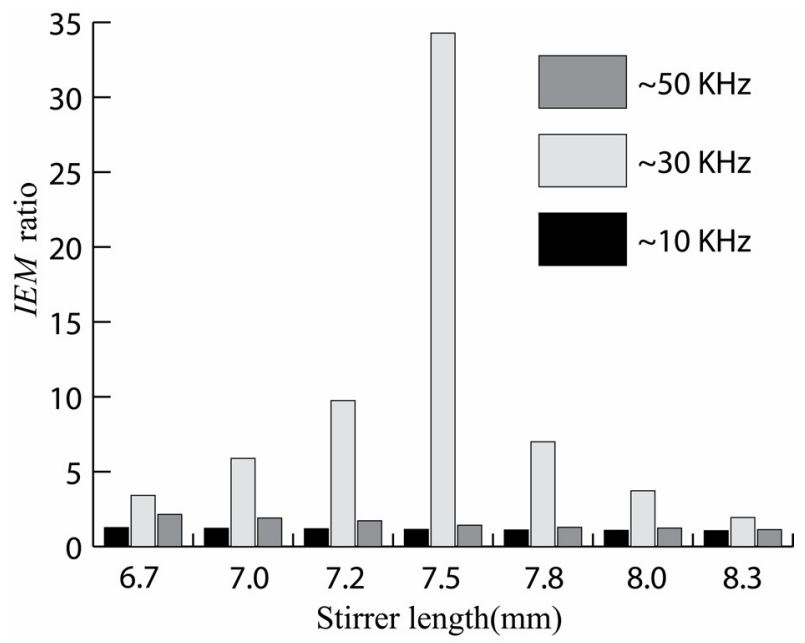

Fig.6 IEM ratios as a function of the stirrer length. 


\section{Optimal Length Searching Algorithm}

Because of demands to different length of stirrer and catheter for various thrombus and people, the stirrer length and catheter are the important parameters in design. In this paper, suppose the length of stirrer is given based on the practical demands. Further the angle of groove in the stirrer (Fig. 2) is set at 80 degree, which is an optimum value, to from the maximum vertical amplitude at stirrer tip based on our previous research ${ }^{(11)}$. An optimal total length searching algorithm is then proposed on the simple theoretical estimation and numerical simulation.

\subsection{Simple Theoretical Estimation}

Consider the micro-stirrer as a cantilever beam (Fig. 7 case (a)). The longitudinal resonant frequency of simple beam can be estimated simply by

$$
f_{l}=\frac{(2 n-1)}{4 L_{T}} \sqrt{\frac{E}{\rho}}
$$

where $n$ is mode shape number.

Further, as shown in Fig. 7 case (b) the stirrer can be assumed as a separate simple beam with one end clamped and one end free (cantilever configuration). Therefore, the natural frequency at the first bending mode is given by

$$
f_{b}=\frac{\gamma_{b}^{2} h}{4 \sqrt{3} \pi L_{T}^{2}} \sqrt{\frac{E}{\rho}}
$$

For a fixed-free simple beam the specific constant of the first mode, $\gamma_{b}$, is 4.694 .

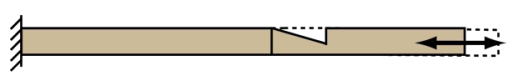

Resonance frequency of longitudinal vibration mode of models $=f$,

Expecting mode shape

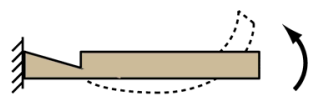

Resonance frequency of bending vibration mode of stirrer $=f_{b}$

Fig. 7 Generation principle of expected mode shape.

Based on amount of numerical experiment data, it is found that if the total length $L_{\mathrm{T}}$ is selected in a way that frequency $f_{l}$ is approximate to $f_{b}$, the possibility to find the matching lengths in the stirrer and catheter becomes higher (Fig. 7 case (c)).

$$
f_{b}=f_{l}
$$

In our optimal length searching algorithm, the bending mode frequency $f_{b}$ is used as the starting point in searching program.

\subsection{Searching Algorithm}

The first step in the design is choosing the stirrer length $\left(L_{s}\right)$ with considering the dimension of arterial vessel. And then the $2^{\text {nd }}$ mode of bending natural frequency for the simple beam (cantilever configuration) is calculated by Eq. (3).

As the first approximation assume that the longitudinal resonance frequency is the same as the bending frequency. So the total length of micro-stirrer $\left(L_{T}\right)$ is expressed by the Eq. (5) as a function of the bending frequency. While $n$ is assumed as $2^{\text {nd }}$ longitudinal mode shape.

$$
L_{T}=\frac{(2 n-1)}{4 f_{b}} \sqrt{\frac{E}{\rho}}
$$


Next the modal analysis on the catheter-stirrer is carried out with the total length $L_{\mathrm{T}}$ and stirrer length $L_{\mathrm{S}}$ obtained in above process. Calculate IEM ratios from Eq.(1) for each mode shape at the corresponding resonance frequency $\left(f_{i}\right)$ obtained in the modal analysis. If the maximum value of IEM ratios is bigger than the desired value (Ex., 10) the total length $L_{T}$ and stirrer length $L_{s}$ are the optimum ones. If the $I E M$ ratios are smaller than the desired value, select the frequency $\left(f_{\text {max }}\right)$ for which the IEM ratio is in highest value. Substitute $\left(f_{\text {max }}\right)$ to Eq. $(5)$ again to calculate the $L_{\mathrm{T}}$. Flow the above procedure repeatedly until the highest IEM ratio becomes higher than the desired value. In order to present the procedure clearly, the flowchart of the optimal length searching algorithm is summarized in Fig. 8.

\section{Evaluation of Algorithm}

In order to evaluate and validate the algorithm, several lengths of the stirrer were examined to find the optimum total length $L_{\mathrm{T}}$ which satisfies the expected mode shape. The lengths of stirrer in test are selected arbitrarily as $7.2 \mathrm{~mm}, 8.2 \mathrm{~mm}$ and $14.2 \mathrm{~mm}$. The IEM ratios corresponding to the first three biggest values at each stirrer length are presented in Fig. 9

The appropriate total lengths for each stirrer length were obtained by mode analysis as $65 \mathrm{~mm}, 91 \mathrm{~mm}$ and $312.5 \mathrm{~mm}$ respectively. The results are also shown that the specific designed lengths have surely generated the expected mode shape as the one showed in Fig. 4 case (b).

Furthermore, the harmonic analysis was carried out on each of them to determine the driving frequencies which excited the maximum tip deflection. It is noticeable that the frequency at the desired mode shape is related to the stirrer length. As illustrated in Fig. 9 the excited frequencies for each desired mode shape are $53470 \mathrm{~Hz}, 38915 \mathrm{~Hz}$ and $10900 \mathrm{~Hz}$ respected to the stirrer lengths of $7.2 \mathrm{~mm}, 8.2 \mathrm{~mm}$ and $14.2 \mathrm{~mm}$. In practical case sometimes the excited frequency is also very important for effective dissolution of the thrombus. The above result also hints that the total length can be also determined for a given exciting frequency based on the above proposed searching algorithm.
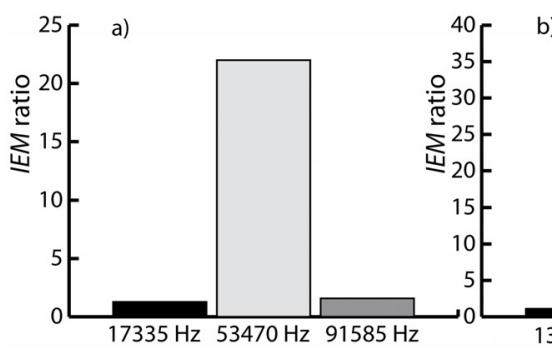

b)

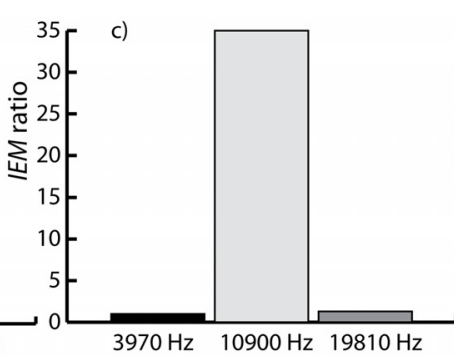

Fig.9 IEM Ratios obtained at frequencies with the first three highest peaks of vertical amplitude at tip. a) Total length $65 \mathrm{~mm}$ - Stirrer Length $7.2 \mathrm{~mm}$, b) Total length $91 \mathrm{~mm}$ - Stirrer Length $8.2 \mathrm{~mm}$

c) Total length $312.5 \mathrm{~mm}$ - Stirrer Length $14.2 \mathrm{~mm}$ 


\section{Conclusion}

The present study introduced a novel micro-stirrer, which is applicable in thrombus treatment. The tip trajectory of the expected mode shape was worked more stability and reliable than the other mode shapes. The ratio of vertical deflection amplitude on tip to that on catheter was the most highest for expected mode shape. This IEM ratio was used for comparing and evaluating the quality of the obtained results from the proposed design methods. In fact, the design processes is a loop of finding lengths which matching the longitudinal resonance frequency of the catheter and bending resonance frequency of the stirrer. It seems that stirring in different frequencies is achievable by just properly adjusting the lengths due to it doesn't dictate by the restriction in a blood vessel diameter.

\section{References}

(1) Tsirka, S.E., Clinical Implications of the Involvement of tPA in Neuronal Death. J. Mol. Med. Vol.75 (1997), pp.341-347.

(2) Gingrich, M.B. and Traynelis, S.F. Serine Proteases and Brain Damage - Is There a Link?, Tr. Neurosci, Vol.23 (2000), pp. 399-407

(3) Flint, A., Duckwiler, G., Budzik, R., Liebeskind, D., Smith, W., Mechanical Thrombectomy of Intracranial Internal Carotid Occlusion: Pooled Results of The MERCI and Multi MERCI Part I Trials. Stroke, Vol.38, No.4 (2007), pp.1274-80

(4) Smith W., Sung G., Starkman S., et al., Safety and Efficacy of Mechanical Embolectomy in Acute Ischemic Stroke: Results of The MERCI Trial. Stroke, Vol.36, No.7 (2005), pp.1432-8

(5) Masahiro H., Michiya S., Yasuhiro U., Katsunori M., Ken Y., and Yoshiro T., Electrically Controlled Piezoelectric Motion of Piezoelectric Chiral Polymeric Fibers, Japanese Journal of Applied Physics, Vol.47, No.9 (2008), pp. 7642-7645

(6) Tachibana K, Tachibana S, Prototype Therapeutic Ultrasound Emitting Catheter for Accelerating Thrombolysis. J Ultrasound Med, Vol16, No.8 (1997), pp.529-535

(7) Alexandrov V., Demchuk M., Felberg A., Christoh I, Barber A., Burgin W, Malkoff M., Wojner W. and Grotta C., High Rate of Complete Recanalization and Dramatic tPA Infusion When Continuously Monitored with 2-Mhz Transcranial Doppler Monitoring, Stroke, Vol.31, No.3 (2000), pp.610-614

(8) Jiang, Z., Morita, M., Watanabe, T., Kato, S. and Suzuki, M. Study on In-vivo Measurement of Solubility for Cerebral Thrombus Dissolution, International journal of Applied Electromagnetic and Mechanics, Vol.24, (2006), pp. 225-234.

(9) Krasokha, N., Theisen, W., Reese, S., Mordasini, P., Brekenfeld, C.,Gralla, J., Slotboom, J., Schrott, G. and Monstadt, H. Mechanical Properties of Blood Clots - a New Test Method, Materials Science and Engineering Technology. Vol.41, No. 12 (2010), pp. 1019-1024.

(10) M. Morita, Z. Jiang, T. Watanabe, M. Suzuki and S. Kato, Study on Development About Micro-Stirrer for Cerebral Thrombus Dissolution Which Has Sensor Ability, Transactions of the Japan Society of Mechanical Engineers, Series C, Vol.74,No.743 (2008), pp.1798-1803.

(11) M. Morita, Z. Jiang, T. Watanabe, N. Chijimatsu, S. Kato, M. Suzuki, Study on Design of Micro-stirrer for Thrombus Dissolution, Microsyst Technol, Vol.14, (2008), pp. 159-165.

(12) Ajoudanian, M., Jiang, Z. and Morita, M., Analysis and Modeling of Vibratable endeffector Angle of Catheter for Thrombus Dissolution, Proceeding of ASME 2010 Conference on Smart Materials, Adaptive Structures and Intelligent Systems, Vol.2 (2010-9), pp. 349-353 